\title{
DECISION TREE BASED OCCLUSION DETECTION IN FACE RECOGNITION AND ESTIMATION OF HUMAN AGE USING BACK PROPAGATION NEURAL NETWORK
}

\author{
${ }^{1}$ P. Karthigayani and ${ }^{2}$ S. Sridhar \\ ${ }^{1}$ Sathyabama University, Chennai, Tamil Nadu, India \\ ${ }^{2}$ R.M Engineering College, Bangalore, India
}

Received 2013-10-03; Accepted 2013-11-02; Revised 2013-11-12

\begin{abstract}
Occlusion detection in face verification is an essential problem that has not widely addressed. In this study the research is deals about occlusion detection in face recognition and estimation of human age using image processing. The objects hide from another object is called as occlusion. Occlusion conditions may vary from face wearing sunglasses, wearing of scarf in the eyes and mouth positions. The proposed work consists four stages. Initial stage is to extract the features using canny edge detection technique and to classify the occluded and non occluded region using Decision Tree Based Occlusion Detection (DTOD) classifier. Secondly the face verification and recognition is carried out using Elastic Matching Pattern (EMP) and Maximum Likelihood Classifier (MLC). Back Propagation Neural Network (BPNN) can be used to estimate the age of the human in the third stage. Our experiments are conducted on the database images for the first stage. By considering the first stage the various performance measures of the classifiers are analyzed. The correctly classified instances rate are high compared with the existing classifiers like random forest and bayes classifier. Experiments are conducted using ORL dataset for the second and the third stage. On the basis of the results obtained from the second stage we observed that the face verification was completed with $95 \%$ of accuracy. In the third stage, the age estimation using BPNN algorithm shows better performance results compared with the existing neural network algorithm.
\end{abstract}

Keywords: DTOD Classifier, Elastic Matching Pattern, Maximum Likelihood Classifier, Back Propagation Neural Network, Face Verification, Age Estimation

\section{INTRODUCTION}

Nowadays a major work from the research community is dedicated to occlusion detection in face verification and age estimation of a human. Human face is robust as it changes within a short span of time. Three kinds of approach exists for classification and recognition. First, the Morphological approach, the idea and technique for the analysis and processing of geometrical structures based on set theory and random functions. The goal of morphological processing is to eliminate the imperfections added during segmentation. Secondly, the appearance based approach, the technique for analysis and processing based on the features of the face which can be covering mask or sunglasses. Finally the tree formation for occluded and non occluded region of the face using decision tree induction approach. The features are extracted in various ways like, PCA with eigen faces, local binary pattern, gabor filter, discrete wavelet transform and hough transform. The decision tree C4.3 with the AdaBoost techniques are combined to produce the decision Tree C5.0 algorithm. Ichikawa et al. (2008) have implemented the usage of decision tree C4.3 algorithm, to classify the occluded and non occluded part of the facial image. Ling et al. (2010) have discussed a discriminative approach to fully capture the face features, Support Vector Machine (SVM) and Gradient Orientation Pyramid (GOP) to be applied for face

Corresponding Author: P. Karthigayani, Sathyabama University, Chennai, Tamil Nadu, India 
recognition. The quality of photos, glass appearance, Facial hair changes were taken as the features of age recognition. The euclidean distance was calculated and was taken into account for face recognition. The proposed work overcome the problem phased in those two papers mentioned above. The parameters of the proposed work are illumination, variability in facial expressions, the presence of occlusions for eye glass and beards finally the background. The decision tree C5.0 algorithm is used in the proposed work implementation for occlusion detection in facial image. The proposed work features are extracted using LBP method. The gain ratio is calculated for all the feature attributes by the training vector. The information gain is found out with the information and conditional entropy in the collection of attributes. Entropy of the image is calculated from the information gain in the decision tree C5.0 algorithm.

\subsection{Related Work and Motivation}

Mahalingam and Kambhamettu (2010) have explained within their work concerning the AdaBoost techniques in the face recognition application. Ichikawa et al. (2008) they classify the image from face and non face by the usage of decision tree 4.3 with AdaBoost and LDA techniques. The LDA can be used to integrate all features and finally conclude that eye and mouth has been occluded. The left and right eye, left and right nose, mouth were the features. Ling et al. (2010) have implemented a discriminative approach to capture the face features, Support Vector Machine (SVM) and Gradient Orientation Pyramid (GOP) to be applied for face recognition. The quality of photos, glass appearance, facial hair changes were taken as the features of age recognition. The euclidean distance was calculated and was taken into account for face recognition.

Jaiswal et al. (2011) extracted features using PCA. Also the eigen face, eigen vector, covariance matrix are calculated. Eigen value was used to rank the eigen vectors. Eigen vectors was utilized in characterizing the differences among the images. Euclidean distance was calculated using the difference with the image. To classify the face and non face image the PCA and Eigen method was used. Based on the results they suggested that the future work will give attention to the conditions like head size, orientation, background conditions change. Belhumeur et al. (1997) have calculated the best eigen vectors from large number of eigen values. (Pentlant et al., 1994) have got the features eigen eye, eigen mouth and eigen nose were extracted using modular eigen face method. Twenty images for training and 10 images for testing were taken for the process.

Liao et al. (2006) have focused that, feature extraction is performed by the local binary pattern approach for various intensity and gradient values. The texture features could be identified using the Tsallis entropy values. Ramesha et al. (2010) have extracted the global and grid features, age classification was carried out by wrinkles as the feature. The gender identification was done by posteriori, priori probability values. The classifier classified the young aged, middle aged and old aged by back propagation neural network. The Feature Extraction based Gender Identification and Age Classification (FEBGIAC) algorithm was used to recognize the face, Gender and Age estimation. Hewahi et al. (2010) the age estimation was carried out by neural network with the classification of age like, childhood, young, youth and old. Tan et al. (2008) have focused on the standard local binary pattern method was applied to extract the feature and elastic matching pattern was used to recognize the face. Ling et al. (2007) have considered the skin color as the feature. Based on the hemoglobin and melanin values the face was recognized. Geng et al. (2007) they proposed an automatic age estimation method named Aging Pattern Subspace (AGES). The sequence of a particular individual's face images sorted in time order. To reconstruct the face image with minimum reconstruction error, the position of the face image in that aging pattern will then indicate the age. Khan et al. (2013) have deals about the gender classification using decision tree C5.0 algorithm with the help of Weka 3.6.4 tool for data mining.

Karthigayani and Sridhar (2011a; 2011b; 2010; 2013) have proposed and implemented the various face recognition system using local binary pattern, elastic matching pattern, Discriminative approach. They concluded that the occlusion detection using decision tree C5.0 algorithm is one of the method to classify the occluded and non occluded facial image under occlusion conditions. Based on the literature survey, it is found that, there is no systematic approach for the occluded conditions for the face image. Hence the proposed method is mainly focused on Decision Tree C5.0 induction method.

\subsection{Proposed Architecture}

Architecture describes the fundamental organization of a system with their components and relationships to other environment. Architecture is the set of decisions that can be made before specific applications designed. 
Architecture can divide the system also it divides the roles and responsibilities of work that can be effectively managed.

The flow diagram of the proposed work shown in Fig. 1 Classification is carried out using Decision Tree C5.0 algorithm. The Face is recognized using elastic matching pattern and also with maximum likelihood classifier. Last stage, the age can be estimated using the back propagation neural network.

\section{MATERIALS AND METHODS}

The proposed works have overcome the limitations in the existing method. Based on the detailed study the work can be divided into three stages. The stages are as follows:

- First phase is consists of feature extraction using canny edge detection technique and to classify the occluded and non occluded face using Decision Tree C5.0 Occlusion Detection classifier (DTOD Classifier)

- Second phase for face verification using elastic matching pattern and maximum likelihood classifier for recognition

- Third phase include the back propagation neural network to estimate the age of a human

\subsection{First Phase (Feature Extraction and Classification)}

Feature are functions of the original measurement variables that are useful for classification. It is the process of defining a set of features or image characteristics, which will most efficiently or meaningfully represent the information that is important for analysis and classification. Different kinds of feature extraction techniques are, available in image processing. Initially the Geometry based features, Edge Detection techniques, Template based approach, Color segmentation technique and finally the appearance based techniques. The Edge detection technique was used to extract the features of the face in the proposed work implementation. The features are extracted using canny edge detection method. The MATLAB image processing toolbox can be used to find the feature extraction.

The below Fig. 2 shows the canny edge detection techiques using MATLAB 7.9.
The features are extracted using Fig. 2 using canny edge detection technique and the output from feature extraction taken into account for the next process. The occluded and non-occluded feature attributes extracted and sent to the input for the next process.

The decision tree based occlusion detection classifier is used to detect the occluded and non occluded part of the input image. Classification is carried out for full face as a feature.

Decision tree model is based on the training set and the values in the feature attributes. The decision tree is a powerful and popular tool for classification and prediction. Decision tree is the form of classifier in the form of tree structure. Decision node specifies a test on a single attribute and leaf node indicates, value of the target attribute. The edge is a split of one attribute and the path is a disjunction of test to make the final decision. In Fig. 3 The DTOD classifier is based on the Decision Tree C5.0 algorithm. From the Fig. 3 considering the full face as a feature, classification for occluded and non occluded part of the face has been identified using Decision Tree C5.0 algorithm. The Decision Tree C4.5 with AdaBoost techniques are combined to produce the Decision Tree C5.0 algorithm. The Decision Tree C5.0 algorithm is applied in the proposed work implementation. The Gain ratio is calculated for the training set attributes for all the features. Information Entropy is defined as:

$$
E(S)=-\sum_{i=0}^{m} P_{i} \log _{2} P_{i}
$$

where, $\mathrm{S}$ is the test data $\mathrm{n}$ is the sample set. $\mathrm{I}=1,2,3$ ...m. $\mathrm{P}_{\mathrm{i}} \mathrm{P}_{\mathrm{ij}}$ are the proportions:

$$
\begin{aligned}
& \mathrm{P}_{\mathrm{i}}=\mathrm{n}_{\mathrm{i}} /[\mathrm{S}] \\
& \mathrm{P}_{\mathrm{ij}}=\mathrm{n}_{\mathrm{ij}} /[\mathrm{Sj}]
\end{aligned}
$$

\subsection{DTOD (Decision Tree Classification Approach) Classifier}

The decision tree C5.0 with J48 classifier are the enhanced version of C4.5 algorithm. It was designed by Quinlan Ross in 1993. The Decision Tree Based Occlusion Detection Classifier (DTOD) is performed using MATLAB 7.9 with different feature attributes. The attributes distance can be carried out using Euclidean distance. 


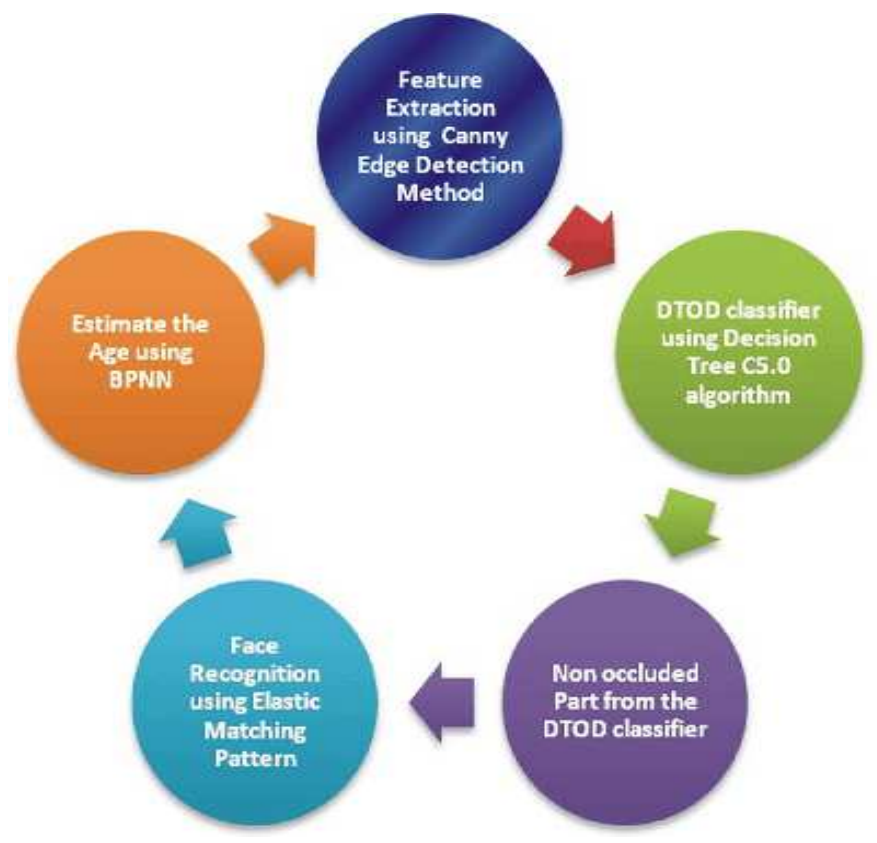

Fig. 1. Architecture of the proposed work

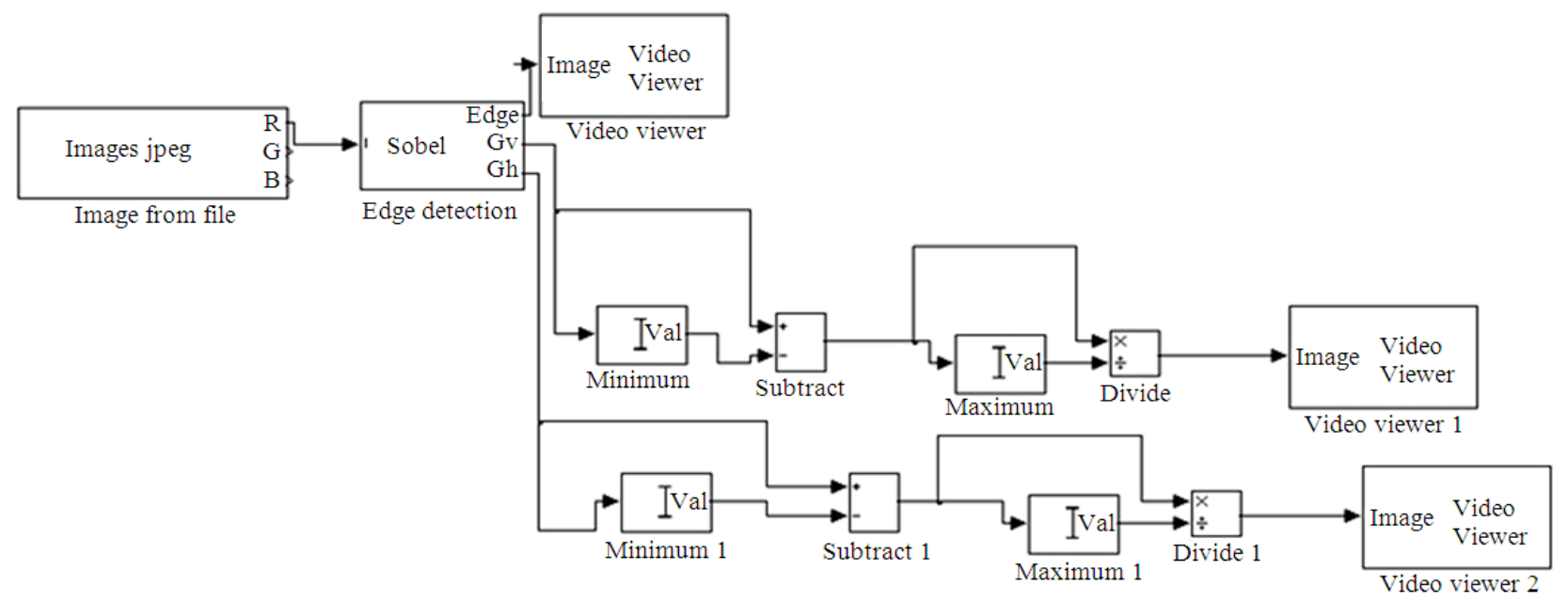

Fig. 2. Architecture of feature extraction using canny edge detection technique

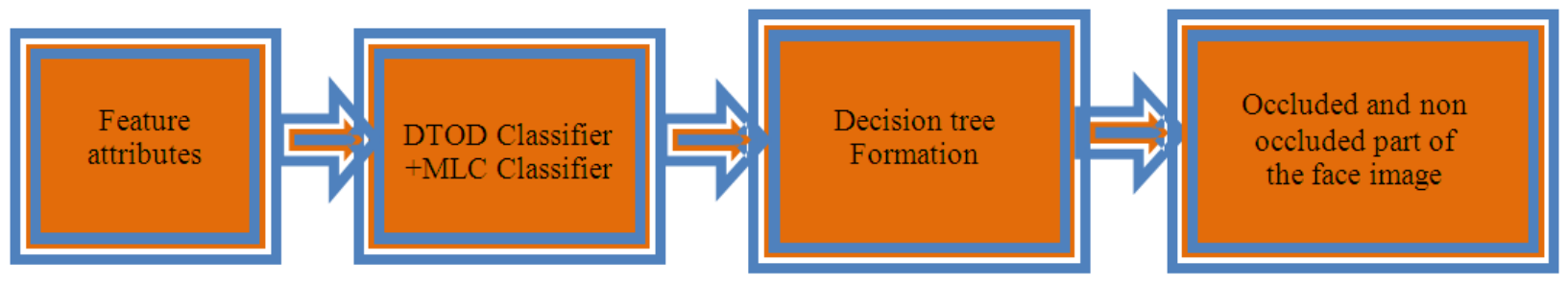

Fig. 3. Architecture of the second phase 
A decision tree is a top down architecture in hierarchical nature where the branch node indicates the two possible ways of process and the leaf node indicates the decision. The leaf node indicates the accept or rejection condition. The feature attributes from the input image, the classifier output and defining a rule set are the main characteristics of the decision tree classification approach.

Conditional Entropy is defined as:

$$
E(S / A)=\sum_{j=1}^{v} P_{j}^{!} \sum_{i=1}^{m} P_{i j} \log _{2} P_{i j}
$$

The information Gain is found out with the information and conditional entropy (By the reference from (Mahalingam and Kambhamettu, 2010) Information Gain = Information Entropy-Conditional Entropy).

\subsection{Extract the Feature Attributes}

The list of key attributes used in the proposed work experiments are obtained using Edge Detection Technique and Euclidean distance. The attributes are full face, eyes, nose and mouth Table 1.

To train the classifier we use the UCD from Fig. 4 dataset for 95 images wearing sunglass or glasses and also to have beards and mustache. The image size is $96 \times 96$ pixels, the frontal images with variations in pose, illumination and expression. A few sample images are shown below.

\subsection{DTOD Classifier Algorithm}

Acquisition of input image:

- Whole face classifier scans the input image and results the confidence measure

- Individual face part classifier is also scanned and finds the confidence measure

- Integrate step 2 and 3 is used to find a face or non face

- Step 4: the AdaBoost and lda is used for integration

$$
\left\{\begin{array}{cc}
1 & \sum_{\mathrm{i}=0}^{\mathrm{i}=\mathrm{N}-1}\left(\mathrm{e}_{\mathrm{i}} \mathrm{h}_{\mathrm{i}}(\mathrm{x})\right) \geq \mathrm{T} \\
\text { lda stageh }(\mathrm{x})=-1 & \text { otherwise }
\end{array}\right.
$$

Where:

$h_{i}(x)=$ The output value for the $i$ th classifier

$\mathrm{T}=$ Threshold

$\mathrm{e}_{\mathrm{i}}=$ The projection weight

\subsection{Maximum Likelihood Classifier} constant

if (test face distance -minimum distance) <

accept face for non occlusion

else reject face for occlusion

\subsection{Decision Tree Stage}

output from the classifier

lda stage:

if output is $\geq$ threshold or output is < threshold take right eye, right eye will be the root

if output is $\geq 0$ or output is $<0$

right eye: left eye-left

if left eye .output is $\geq 0$ then take lip else occlusion on left

if lip output is $\geq 0$ then take non face else output is $<0$ then

occlusion on mouth

right eye: left eye-right

if left eye. output is $\geq 0$ then occluded on

right side

else output is $<0$ then take nose

if nose.output is $\geq 0$ occlusion on eyes

else non face

The different classifiers are used to find the occlusion and non-occlusion part of the face using WEKA 3.6.4. The decision tree C5.0 (J48) classifier with MLC performs better result compared with other classifiers.

\subsection{Second Phase (Face Verification)}

Face verification is performed by non occluded part of a face image. The occluded part of the face is hided with other objects. So it is not possible to find the verification of the face. The output from the first stage as taken as an input for the second stage. From Fig. 5 the features can be extracted and the face verification is carried out using elastic matching pattern and the recognition is achieved using MLC.

Table 1. Attributes and their description

\begin{tabular}{ll}
\hline List of key attributes & Description \\
\hline FF & Full face \\
SLEP & Start Left Eye Position \\
SREP & Start Right Eye position \\
SLP & Start Lip position \\
SNP & Start Nose Position \\
Classification Flag & Either-Occluded (O) or \\
& Non Occluded (NO) \\
\hline
\end{tabular}



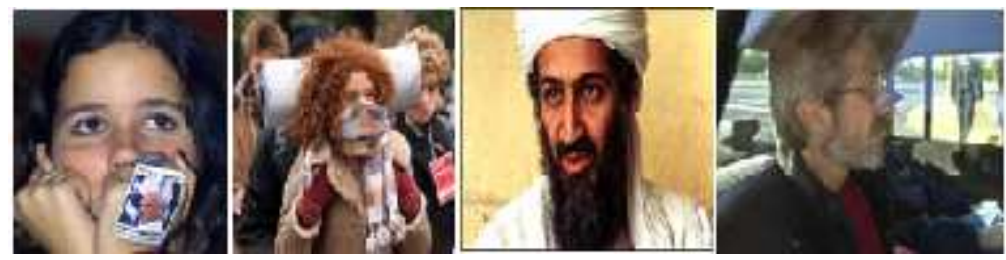

Fig. 4. Various faces in UCD database

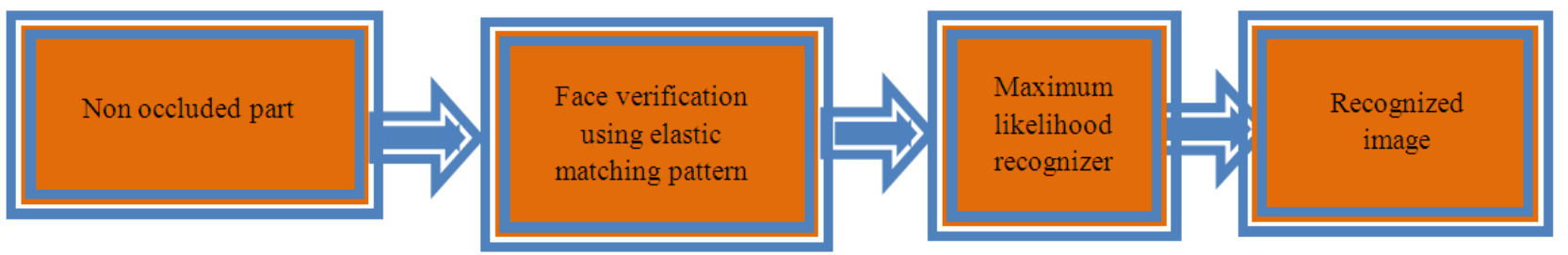

Fig. 5. Face verification and recognition

\subsection{Face Verification by Elastic Matching Pattern}

Human face is a combination of small square structure of blocks. Nearest blocks are overlapped and the image is divided into blocks. First to find the offset for the two adjacent blocks, if the blocks are not equal distance some distortion has happened. The elastic matching is applied in the test image and to seek the neighborhood in the training image. Find the best match i.e., the block with minimum distance to it. The nodes in square as $21 \times 21$ and the block size is $5 \times 5$.

\subsection{Face Recognition Using Maximum Likelihood Classifier}

Model likelihood for all the faces in the training data set have been calculated. Figure 6 shows the block diagram of the MLC. The highest log likelihood is identified and that was representing the face. Euclidean measure is applied to test if the face is in the training set or database. If the log likelihood is in the declared distance then the face is recognized and it exists in the training set:

if (test face distance-minimum distance) < constant accept face

else reject face

\subsection{Third Phase (Age Estimation)}

The proposed method is to identify the smaller age gaps with respect to image quality, spectacles and facial hair change in the existing system. Also Gender was identified using the face boundary estimation method.
The noise was removed in the extracted image and the filters have applied to detect the original image.

\subsection{Face Boundary Estimation}

From the Fig. 7 the edge was calculated after converting the original image into the binary scale image. The face boundary differs from different age groups. After analyzing the image, the image fall in any of the age group. Morphing describes the action taking place when an image (digital image) gets transformed into another or in a more technical way, it can be described as the combination of generalized image warping with cross-dissolve image elements.

\subsection{BPNN (Weight and Threshold)}

The neurons have been identified and the threshold is calculated. The weights are applied for all the week neurons for recognition. The Age gaps for the two age intervals are small, because of identifying the exact age of a human. The week neurons are trained with the additional weights and threshold. The error rate was observed for all the neurons and when high it again enters into the next cycle with additional increasing weight.

The neurons have been identified and the threshold is calculated. The weights are applied for all the week neurons for recognition. The Age gaps for the two age intervals are small, because of identifying the exact age of a human. The week neurons are trained with the additional weights and threshold. The error rate was observed for all the neurons and when high it again enters into the next cycle with additional increasing weight. 


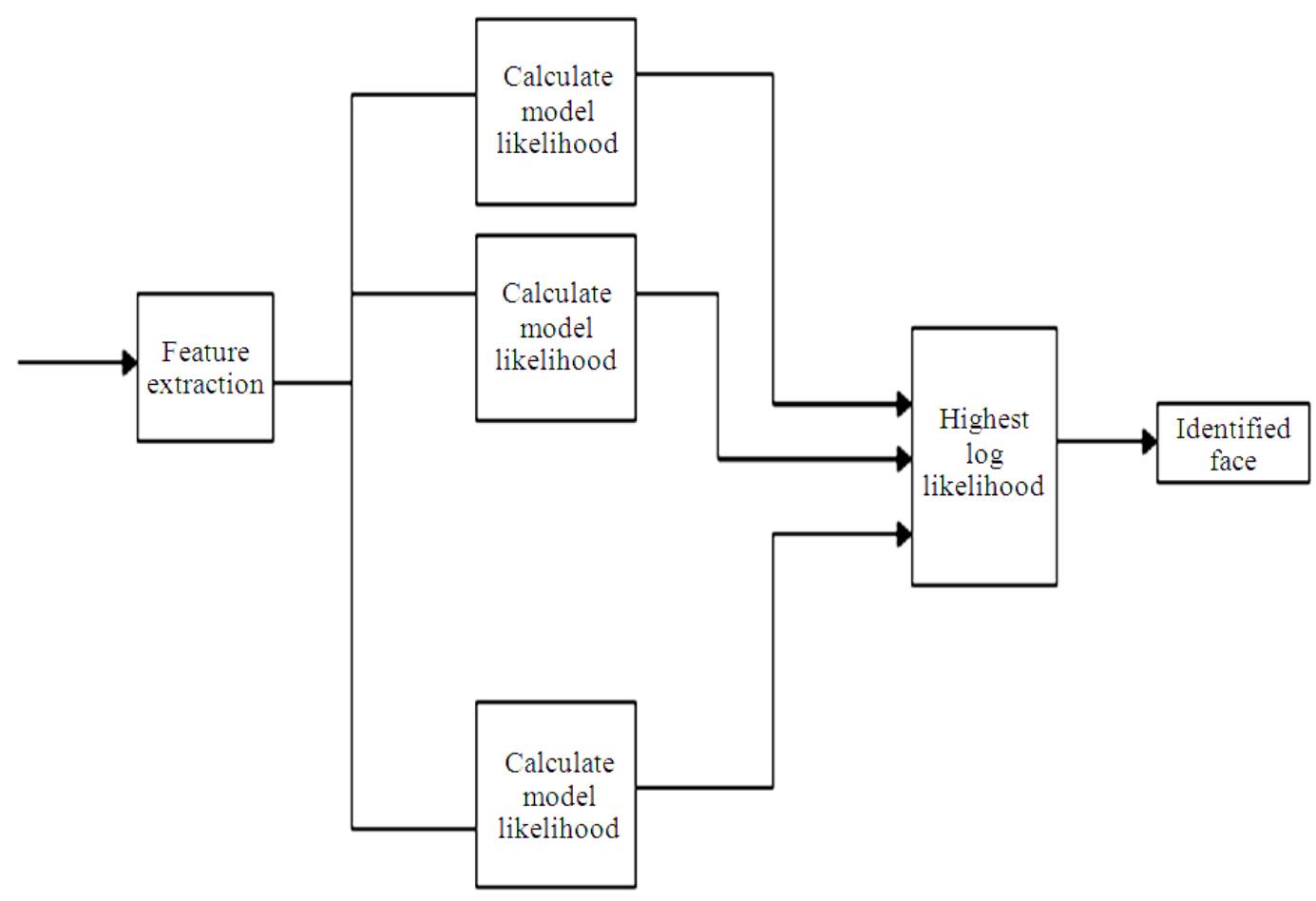

Fig. 6. MLC recognizer

In Fig. 8 young, middle and old age is divided into the smaller age gap. To estimate the age of a human, the smaller age gap is to give more accurate result compared with the larger age gaps.

Based on (Ling et al., 2007) article, the back propagation neural network algorithm is used to implement the proposed work:

Step \#1: Read the test image

Step \#2: Select the training image from the set of images with minimum age gap.

Step \#3: Update the weights and biases of the network.

Step \#4: Propagate the partial derivatives of the error function backward through the network.

Step \#5: Normalize the data

Step \#6: Generate Random test vector

Step \#7: Repeat until stop condition is reached.

Step \#8: Age is calculated using the test image

\subsection{Network Architectures}

Supervised and unsupervised network architecture are used to develop the architecture of the proposed work. The inputs, hidden layer and output are used to model system and classify the noisy data to predict the future events. Feedforward networks, radial basis networks, Dynamic networks and Learning Vector Quantization (LVQ) are the four types of supervised network. The feedforward networks include backpropagation, cascade-forward backpropagation, feedforward input-delay backpropagation.

\subsection{Preprocessing and Postprocessing Functions}

To improve the efficiency of the neural network the preprocessing the network inputs and targets are very important. The neural network toolbox provides the preprocessing and post processing functions. The steps to create a neural networks are:

- The input vectors dimension reduction

- Analysis between the network response and the corresponding targets

- Measure the inputs and targets so that they fall in the particular range

- Find the mean and standard deviation of the training data set

- Create a neural network after preprocessing and data normalization 


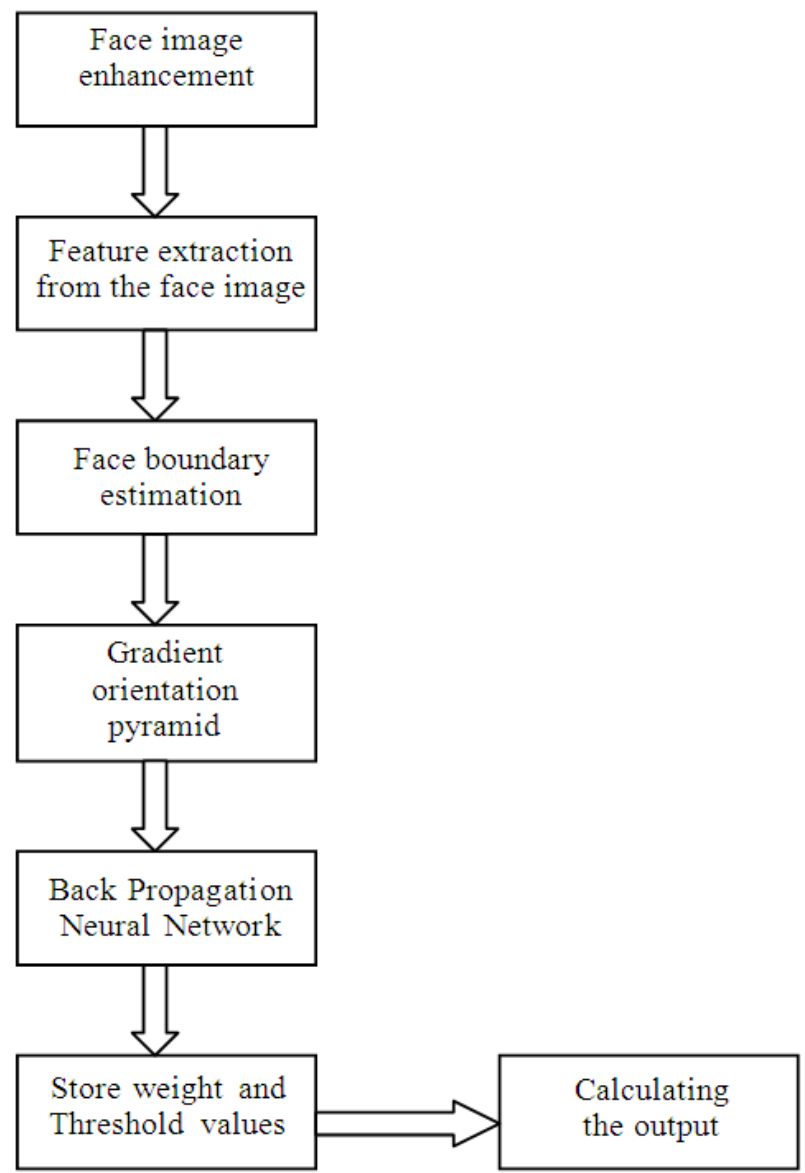

Fig. 7. Age estimation

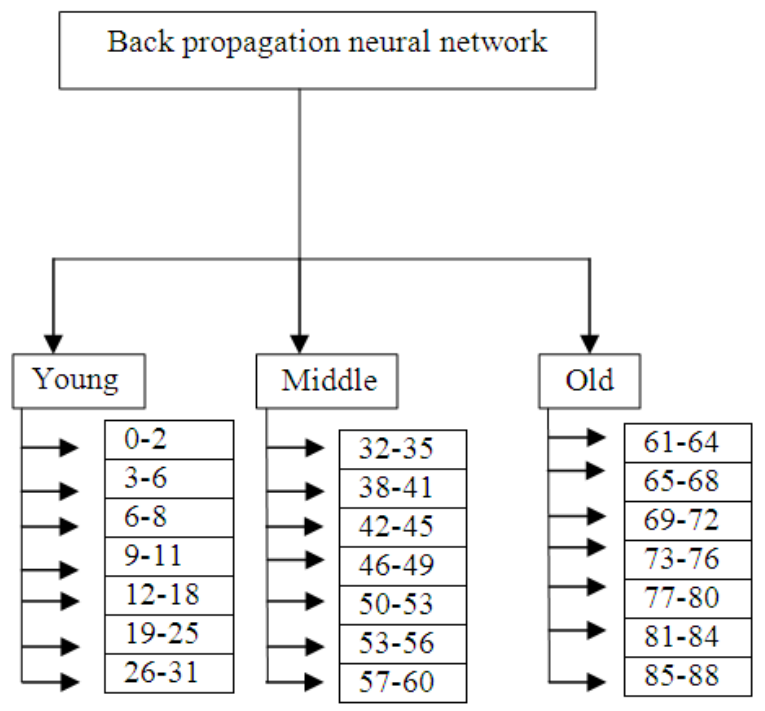

Fig. 8. The age gap for young, middle and aged person 


\subsection{Performance Analysis}

The comparative analysis with different classifiers and the output are analyzed. The decision tree based occlusion detection performed better result compared with the existing work. The comparative study with the existing work for age estimation done with the neural network model. The BPNN shows better performance result than the existing one.

\section{RESULTS}

The feature extraction can be done using canny edge detection and the Euclidean distance values are calculated using image processing tool box in MATLAB software. The output from the Model output for feature extraction was shown in Fig. 9.

Figure 10 described the output for classification from occluded and non occluded part of the face. Figure 11 described the decision tree structure for the occluded part in the face image using decision tree C5.0 algorithm.

Table 2 describes the rule set for classify the occluded part with the non occluded part of the face of a human.

Figure 11 described the face verification using MLC and the age of a human using Back Propagation Neural Network.

Table 2. Classification rule set

\begin{tabular}{lll}
\hline Rule \# & Classification Rule set & Classification \\
\hline 1 & If $(\operatorname{snp}<2.45)$ and if $(\operatorname{slp}>0)$ & No Occlusion (NO) \\
2 & If $(\operatorname{snp}>2.45)$ and if $(\operatorname{slp}>=1.75)$ & NO occlusion (NO) \\
3 & If $(\operatorname{snp}>2.45)$ and if $(\operatorname{slp}<=1.75)$ and if & O (Occlusion on left mouth) \\
& $(\operatorname{snp}<4.95)$ and if $(\operatorname{slp}<1.85)$ & \\
\hline
\end{tabular}

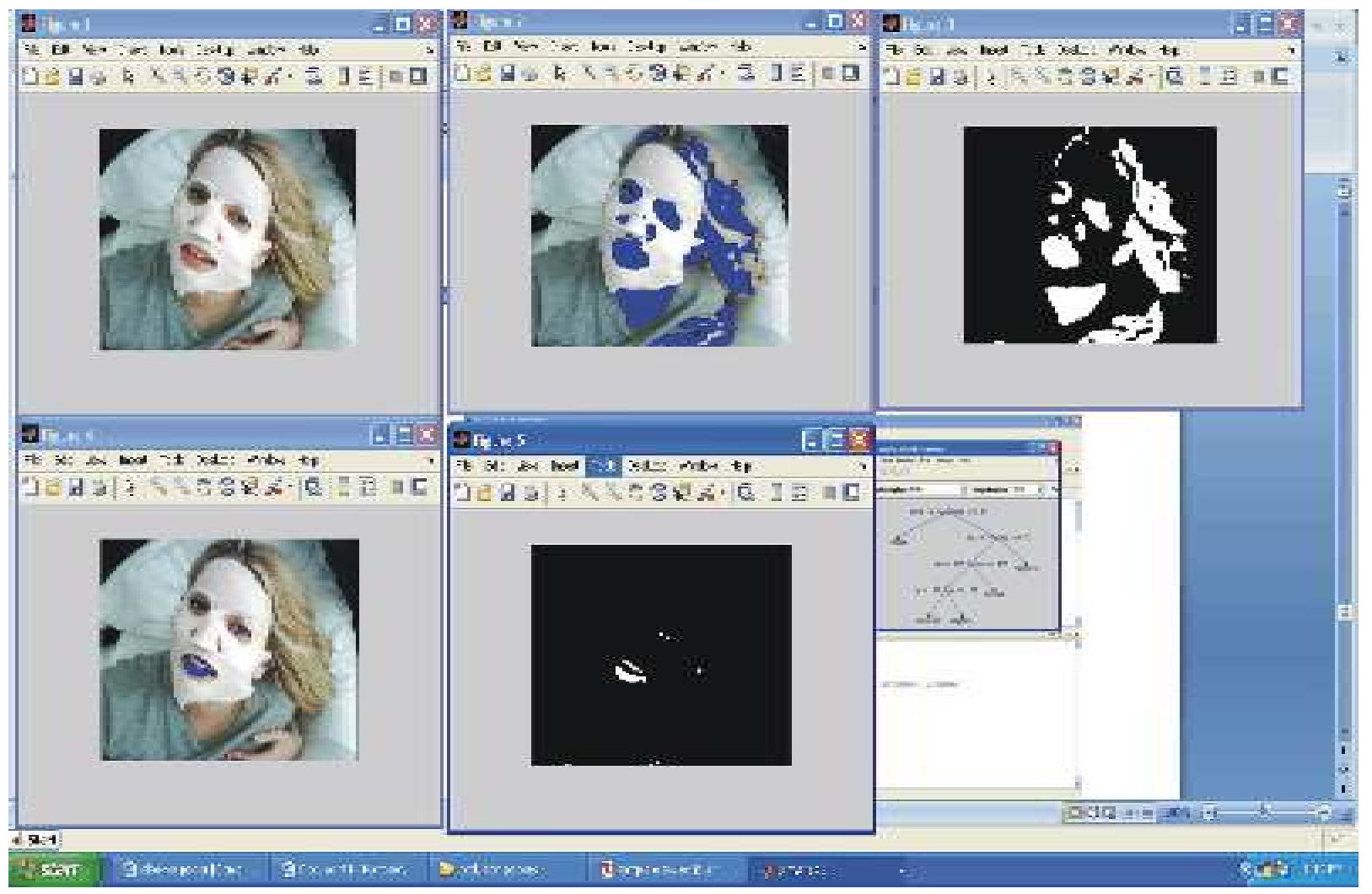

Fig. 9. Feature extraction using canny edge detection technique 


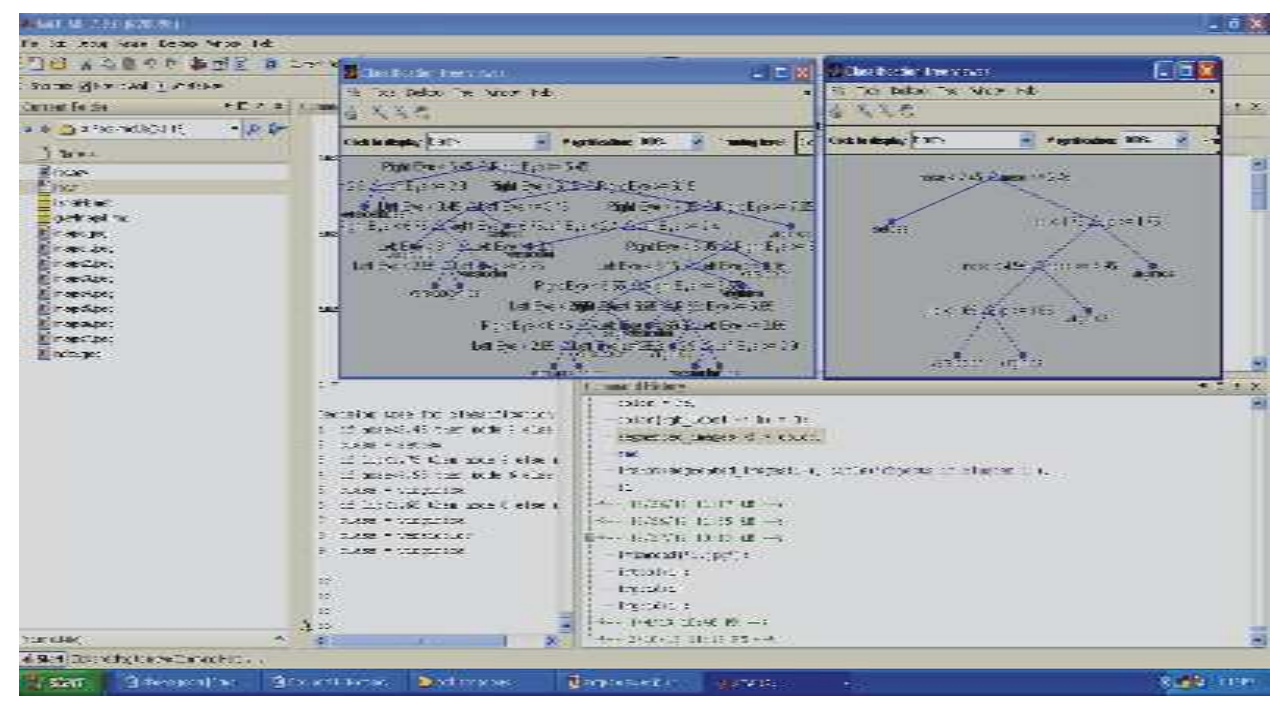

Fig. 10. Occlusion detection using decision tree C5.0 algorithm

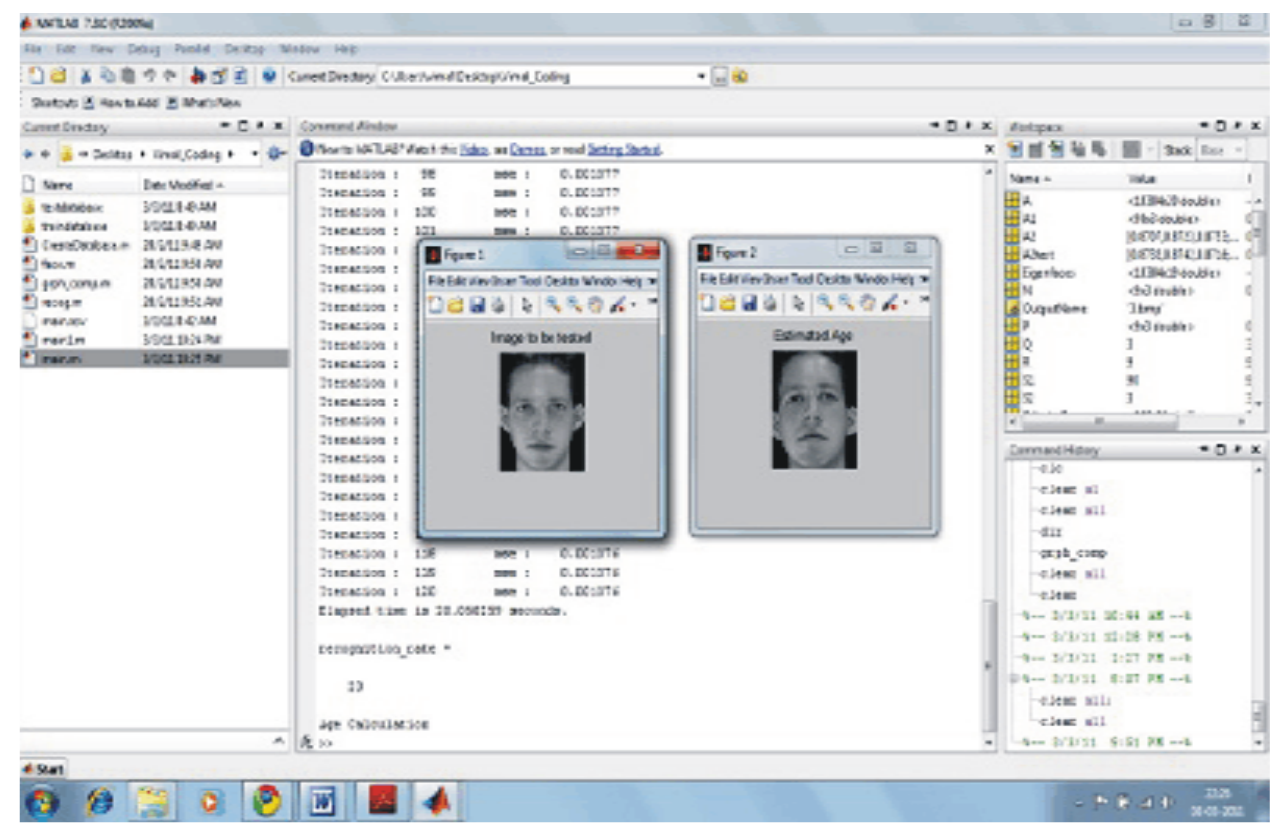

Fig. 11. Face verification using MLC

\section{DISCUSSION}

From Fig. 12 describes the age of a human and Fig. 13 represents the number of neurons in the hidden layer and the execution time in seconds. Figure 14 shows the comparative analysis carried out for neural network and Back propagation neural network. The BPNN shows better performance compared with the existing one.

The Table 3 describes the different classifiers and the classified instances rate. The decision tree occlusion detection classifier will give highest classification instances compared with other classifiers. The Incorrectly classified instances are very low in DTOD classifier compared with the remaining classifiers. 
P. Karthigayani and S. Sridhar / Journal of Computer Science 10 (1): 115-127, 2014

Table 3. Classification accuracy of the training set

\begin{tabular}{lllllllr}
\hline & $\begin{array}{l}\text { Correctly } \\
\text { classified } \\
\text { instances }\end{array}$ & $\begin{array}{l}\text { Incorrectly } \\
\text { classified } \\
\text { instances }\end{array}$ & $\begin{array}{l}\text { Kappa } \\
\text { statistics }\end{array}$ & $\begin{array}{l}\text { Mean } \\
\text { absolute } \\
\text { error }\end{array}$ & $\begin{array}{l}\text { Relative } \\
\text { absolute } \\
\text { error }\end{array}$ & $\begin{array}{l}\text { Root Mean } \\
\text { squared } \\
\text { error }\end{array}$ & $\begin{array}{l}\text { Root relative } \\
\text { squared } \\
\text { error }\end{array}$ \\
\hline Adaboost & 83 & 12 & 0.8449 & 0.0627 & 0.123 & 10.8192 & 4.3281 \\
Bayes & 74 & 21 & 1.3400 & 0.0900 & 0.437 & 12.8430 & 34.4320 \\
Random forest & 81 & 14 & 0.9380 & 0.0647 & 0.237 & 11.8132 & 32.4380 \\
DTOD+MLC classifier & 92 & 3 & 0.9449 & 0.0487 & 0.116 & 9.8192 & 31.3379 \\
\hline
\end{tabular}

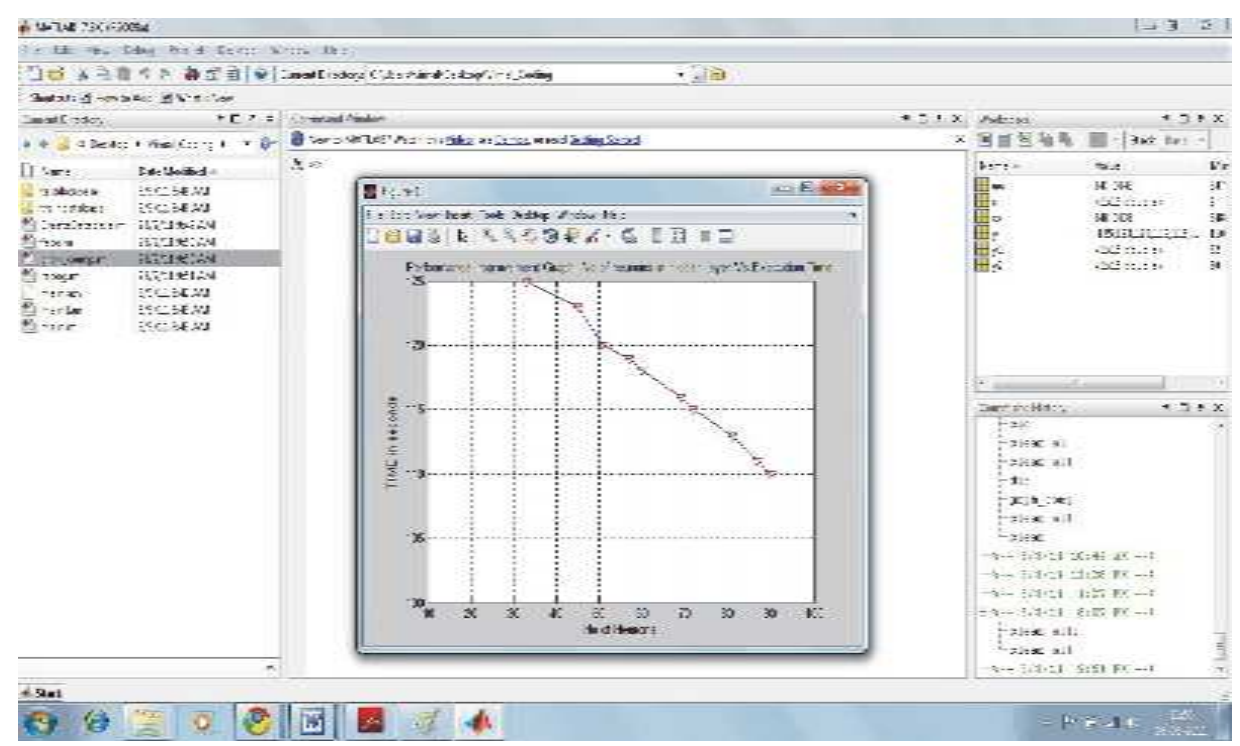

Fig. 12. Performance improvement graph 1(age)

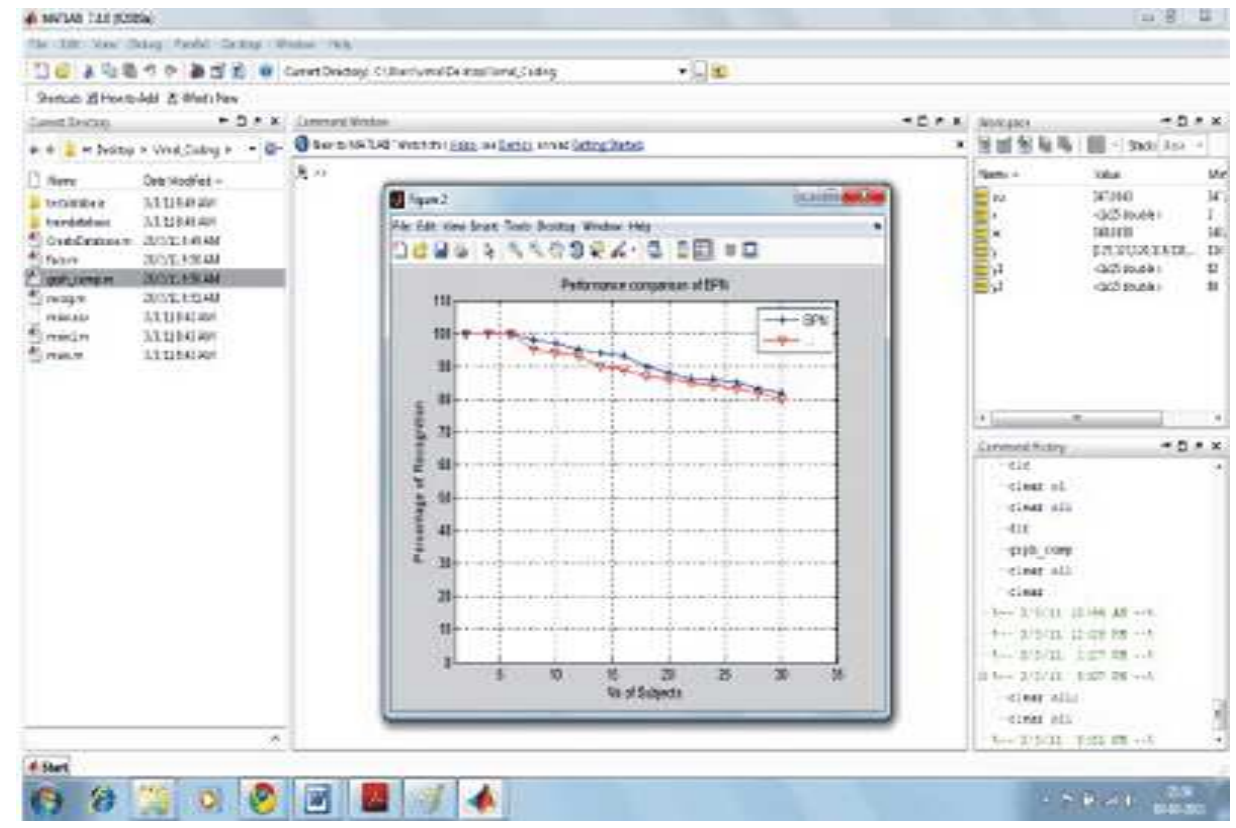

Fig. 13. Performance comparison using BPNN and NN model (age identification) 


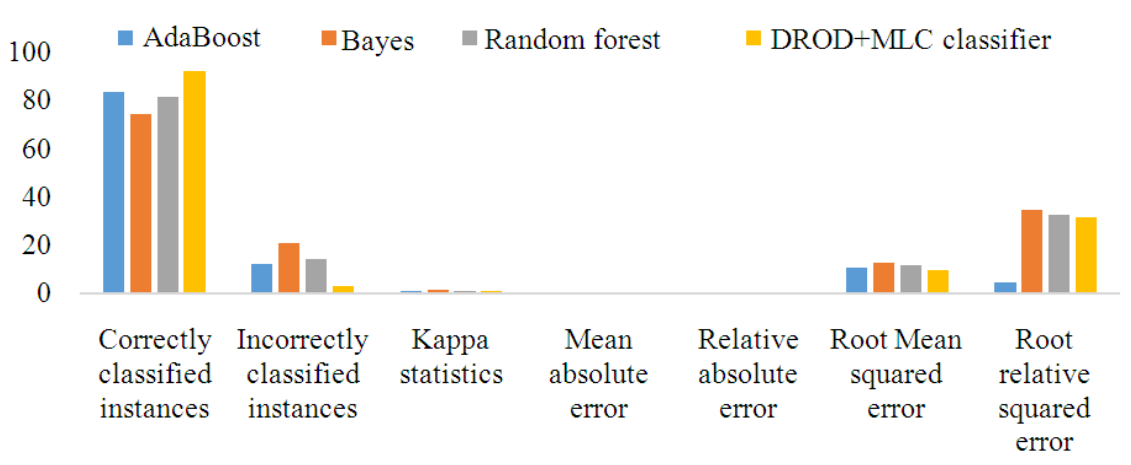

Fig. 14. The performance analysis report

\section{CONCLUSION}

Classification performance using Decision tree C5.0 J48 classifier having high accuracy compared with other classifiers like Bayesian classifier, AdaBoost and Random forest. The face verification done using Maximum likelihood classifier with $95 \%$ of accuracy. The age can be estimated using back propagation neural network. Compared with the neural network the age can be estimated with minimum error rate using BPNN. The future work can be focused on to detect the occluded regions with the help of BFtree, LMT and REP tree sturctures.

\section{REFERENCES}

Belhumeur, P.H., J.P. Hespanha and D. Kriegman, 1997. Eigenfaces Vs. fisherfaces: Recognition using class specific linear projection. IEEE Trans. Patt. Anal. Mach. Intell., 19: 711-720. DOI: 10.1109/34.598228

Geng, X., Z.H. Zhou and K. Smith-Miles, 2007. Automatic age estimation based on facial aging patterns. IEEE Trans. Patt. Anal. Mach. Intell., 29: 2234-2240. DOI: 10.1109/TPAMI.2007.70733

Hewahi, N., A. Olwan, N. Tubeel, S. EL-Asar and Z. Abu-Sultan, 2010. Age estimation based on neural networks using face features. J. Emerg. Trends Comput. Inform. Sci., 1: 301-306.

Ichikawa, K., T. Mita, O. Hori and T. Kobayashi, 2008. Component-based face detection method for various types of occluded faces. Proceedings of the 3rd International Symposium on Communications, Control and Signal Processing, Mar. 12-14, IEEE Xplore Press, St Julians, pp: 538-544. DOI: 10.1109/ISCCSP.2008.4537284
Jaiswal, S., S.S. Bhadauria and R.S. Jadon, 2011. Morphological method, pca and lda with neural networks-face recognition. J. Global Res. Comput. Sci., 2: 35-47.

Karthigayani, P. and S. Sridhar, 2011a. A novel approach for face recognition and age estimation using local binary pattern, discriminative approach using two layered back propagation network. Proceedings of the 3rd International Conference on Trendz in Information Sciences and Computing, Dec. 8-9, IEEE Xplroe Press, Chennai, pp: 11-16. DOI: 10.1109/TISC.2011.6169076

Karthigayani, P. and S. Sridhar, 2011b. A novel approach for face verification and age estimation using local binary pattern, elastic matching and back propagation neural network. Int. J. Intell. Elec. Syst., 5: 37-46.

Karthigayani, P. and S. Sridhar, 2010. Occlusion verification in face detection and age estimation using local binary pattern and DTOD classifier using morph dataset. Proceedings of the International Convention Cum Pre-Conference Workshop on Innovations in Engineering and Technology for Sustainable Development, Sep. 35, pp: 256-263.

Karthigayani, P. and S. Sridhar, 2013. A novel approach for image based occlusion detection in face verification and estimation of human age using back propagation neural network. Sathyabama Universit.

Khan, M.N.A., S.A. Qureshi and N. Riaz, 2013. Gender classification with decision trees. Int. J. Signal Process. Image Process. Patt. Recog., 6: 165-176. 
Liao, S W. Fan, C.S.A. Chung and D.Y. Yeung, 2006. Facial expression recognition using advanced local binary patterns, tsallis entropies and global appearance features. Proceedings of the IEEE Transactions on International Conference on Image Processing, Oct. 8-11, IEEE Xplore Press, Atlanta, GA., pp: 665-668. DOI: 10.1109/ICIP.2006.312418

Ling, H., S. Soatto, N. Ramanathan and D.W. Jacobs, 2007. A study of face recognition as people age. Proceedings of the IEEE 11th Conference on Computer Vision, Oct. 14-21, IEEE Xplore Press, Rio de Janeiro, pp: 1-8. DOI: 10.1109/ICCV.2007.4409069

Ling, H., S. Soatto, N. Ramanathan and D.W. Jacobs, 2010. Face verification across age progression using discriminative methods. IEEE Trans. Inform. Forens. Sec., 5: 82-91. DOI: 10.1109/TIFS.2009.2038751

Mahalingam, G. and C. Kambhamettu, 2010. Face verification with aging using AdaBoost and local binary patterns. Proceedings of the 7th Indian Conference on Computer Vision, Graphics and Image Processing, Dec. 12-15, ACM Press, New York, USA., pp: 101-108. DOI: $10.1145 / 1924559.1924573$
Pentlant, A., B. Moghaddam and T. Starnel, 1994. View-based and modular eigenspaces for face recognition. Proceedings of the IEEE Computer Society Conference on Computer Vision and Pattern Recognition, Jun. 21-23, IEEE Xplore Press, Seattle, WA., pp: 84-91. DOI: 10.1109/CVPR.1994.323814

Ramesha, K., K.B. Raja, K.R. Venugopal and L.M. Patnaik 2010. Feature extraction based face recognition, gender and age classification. Int. J. Comput. Sci. Eng., 2: 14-23.

Tan, N., L. Huang and C. Liu, 2008. Face Recognition based on a single image and local binary pattern. Proceedings of the 2nd International Symposium on Intelligent Information Technology Application, Dec. 20-22, IEEE Xplore Press, Shanghai, pp: 366-370. DOI: 10.1109/IITA.2008.332 